\title{
Relación de Blidingia minima (Ulvales, Chlorophyta) con factores ambientales en Punta Maqueda（golfo San Jorge, Argentina)
}

\author{
Relation between Blidingia minima (Ulvales, Chlorophyta) and environmental factors \\ at Punta Maqueda (San Jorge Gulf, Argentina)
}

\author{
Susana G. Perales ${ }^{1}$ y Alicia L. Boraso ${ }^{1,2}$ \\ ${ }^{1}$ Universidad Nacional de la Patagonia San Juan Bosco. Ciudad Universitaria Km 4, (9000) Comodoro Rivadavia, Provincia del \\ Chubut. Argentina, 2, CONICET \\ ${ }^{2}$ Barrio Diadema, calle Río Negro 160, (9909) Comodoro Rivadavia, Chubut, Argentina \\ sperales@unpata.edu.ar
}

\begin{abstract}
Resumen.- Los factores ambientales relacionados con Blidingia minima (Nägeli ex Kützing) Kylin se estudiaron en Punta Maqueda (golfo San Jorge, Argentina) utilizando análisis de correspondencias, modelos log-lineales para tablas trifactoriales, análisis de redundancia y ANOVA. El grosor del talo está inversamente relacionado con irradiación, temperatura y vientos; la abundancia está directamente relacionada con alta temperatura e irradiación; el tamaño celular, la ramificación y el ancho de los talos se relacionan directamente con agitación del agua y altas temperaturas. Los talos más largos se encontraron en niveles intermareales bajos. En primavera se observaron las mayores biomasas; en verano se recolectaron talos más grandes y en otoño se encontraron céspedes de paredes gruesas con máxima proporción de talos fértiles. La proporción de talos fértiles fue mayor en el nivel más alto del intermareal y mayor en otoño y primavera con relación a verano e invierno, en ambos niveles.
\end{abstract}

Palabras clave: Macroalgas, ecología, Océano Atlántico sur

\begin{abstract}
Environmental factors affecting Blidingia minima (Nägeli ex Kützing) Kylin were studied at Punta Maqueda (San Jorge Gulf, Argentina). Detrended correspondence analysis, redundancy analysis, loglinear models for trifactorial tables and ANOVA were used. Wall thickness is inversely related to temperature, irradiation and winds. Relative abundance is directly related to high temperature and irradiation; branches and cell size relate directly to low surge exposure and high temperature. Longer thalli were found at lower coastal levels. Highest biomass was observed in spring; bigger thalli were collected during summer; in autumn a turf was present with the highest proportion of fertile thalli and thick walled thalli. A greater proportion was found of reproductive thalli at the higher intertidal level, and in autumn and spring in comparison to summer and winter at both intertidal levels.
\end{abstract}

Key words: Macroalgae, ecology, South Atlantic Ocean

\section{Introducción}

Blidingia (Ulvophyceae, Ulvaceae) se diferenció inicialmente de las especies de talo tubular de Ulva L., ubicadas hasta recientemente en el género Enteromorpha Link, por el pequeño tamaño de sus células, la ausencia de procesos rizoidales y la liberación asexual de esporas tetraflageladas, sin estigma ni fototactismo (Bliding 1938, Kylin 1947). Estudios posteriores sobre las especies de este género fueron realizados por Bliding (1963), Garbary \& Barkhouse (1987), Kornmann \& Sahling (1978), Norris (1971), Prange (1978) y Woolcott et al. (2000). La identidad genérica de Blidingia frente a otros géneros de Ulvales es avalada por varios estudios recientes del DNA espaciador ribosomal nuclear trasferido internamente (nuclear ribosomal internal transcribed spacer DNA) (ITS nr DNA) (Hayden et al. 2004). 
La especie tipo, Blidingia minima (Nägeli ex Kützing) Kylin, fue establecida basándose en Enteromorpha minima Nägeli ex Kützing. Esta especie se encuentra en las costas y ambientes estuarinos de casi todo el mundo y se distribuye ampliamente sobre la costa de Argentina (Boraso 1970, 2004).

Estudios sobre aspectos ecológicos han sido realizados por distintos autores (Tittley 1982, Munda \& Markham 1982, Mathieson et al. 1983, Sohn 1983, Kepel 1999). Sin embargo, y a pesar de ser B. minima una especie frecuentemente citada, la información sobre factores que condicionan su distribución y sus características reproductivas, es escasa.

Dado el interés en la ecología y ecofisiología de esta especie (Karsten 1991, Karsten et al. 1992, Laturnus 1996, Larsen \& Sand-Jensen 2006) y su abundancia regional, se consideró oportuno el estudio de la misma en una localidad distante de áreas urbanas y con una amplia diversidad de situaciones ambientales.
Los objetivos de este trabajo fueron: establecer de qué manera los factores ambientales afectan la distribución y morfología de $B$. minima en el intermareal rocoso de Punta Maqueda (golfo San Jorge, Argentina), estimar la variabilidad estacional en abundancia de la especie y conocer el estado reproductivo de la población en el área de estudio.

\section{Materiales y métodos}

\section{Lugar de estudio}

Punta Maqueda se encuentra ubicada en el golfo San Jorge, Provincia de Santa Cruz, Argentina (46 $01^{\prime}$ 's, $67^{\circ} 35^{\prime} \mathrm{W}$ ) y consiste en una playa de areniscas medias a finas con matriz arcillosa (Fig. 1). El régimen de marea es de tipo semidiurno y no desembocan en la zona cursos de agua dulce. Los vientos predominantes son los del cuadrante sudoeste y noreste.

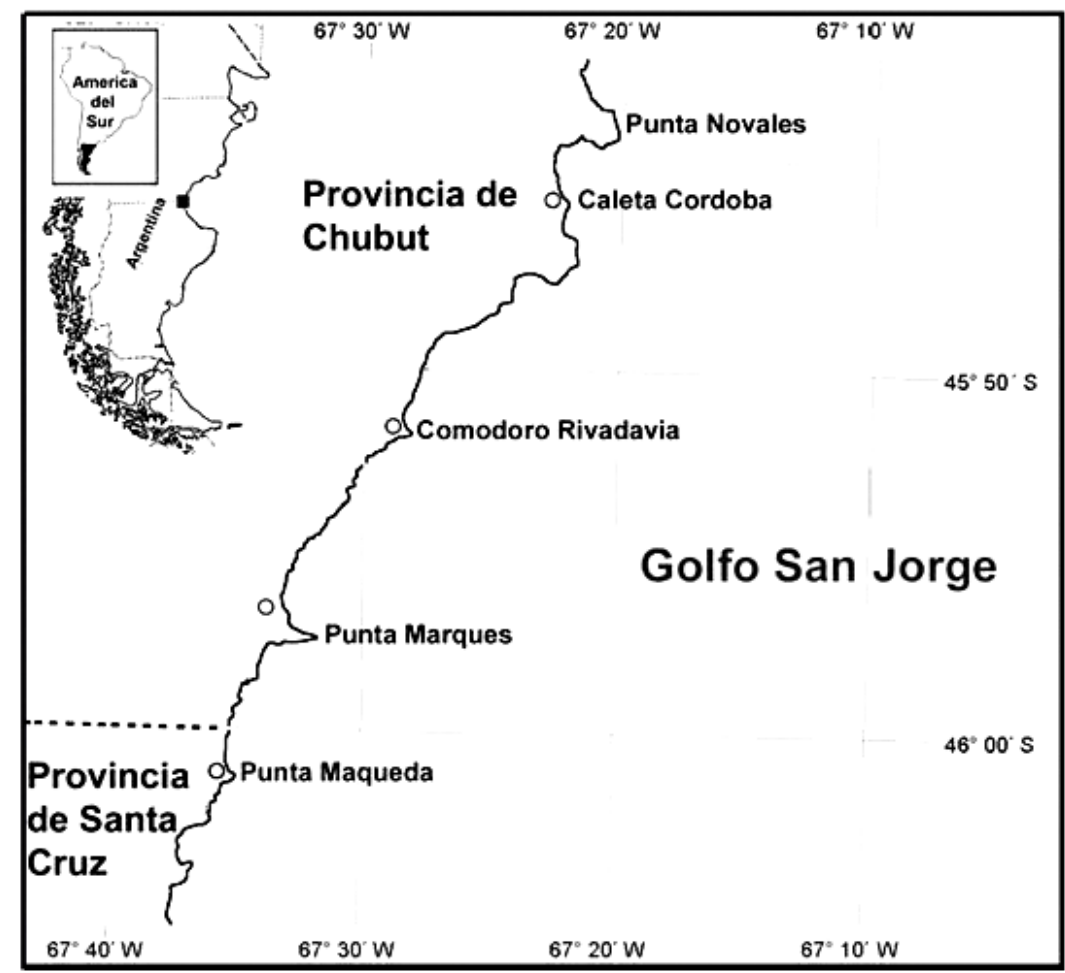

Figura 1

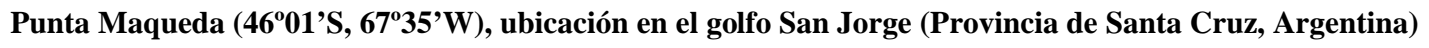

Punta Maqueda (46 $01^{\prime}$ 'S $67^{\circ} 35^{\prime}$ W), location at the San Jorge Gulf, (Province of Santa Cruz, Argentina) 
Se trabajó en dos sitios: el sitio I, al sur de la punta, expuesto al oleaje hacia el SW, y el sitio II, al norte de la punta, expuesto hacia el NE.

El rango de distribución de la especie en la zona fue establecido mediante observaciones estacionales realizadas en el período 1992 - 1996, en el marco de un estudio previo sobre Ulvales de la zona. En base a las mismas, se estableció que $B$. minima se encuentra en el golfo San Jorge generalmente en el intermareal superior rocoso, junto con especies de los géneros Porphyra y Enteromorpha, siendo reemplazada dentro de cuevas por Rhodochorton purpurea (Lightfoot) Woelkerling. Se la puede encontrar también en los niveles intermedios del intermareal, epifita sobre talos senescentes de Porphyra columbina Montagne y Stictosiphonia intricata (Bory) Silva, donde no suele alcanzar más que algunos milímetros de alto.

La intensidad relativa del oleaje fue estimada con la metodología de Doty (1971) y Gerard \& Mann (1979), a través del desgaste de esferas de yeso París; éstas se colocaron pesadas secas a peso constante en tres fechas en los niveles superiores de ambos sitios de estudio (n =3 en cada fecha), transcurridas 48 horas se retiraron y secaron en estufa a $40^{\circ} \mathrm{C}$ hasta peso constante.

Los muestreos biológicos y observaciones ambientales fueron realizados en dos niveles cuyos límites fueron referidos al plano de reducción, utilizando las tablas de marea correspondientes a las fechas de estudio (Servicio de Hidrografía Naval 1998, 1999). Se utilizó como puerto patrón el de Caleta Paula (Caleta Olivia -Provincia de Santa Cruz) y los niveles están expresados como distancia al plano de reducción de las cartas. En el nivel 1 (5,42-3,90 m), los talos forman una cintura continua en el acantilado y en nivel 2 (3,89-3,00 m), los talos crecen también sobre grandes rocas.

En cada uno de los dos sitios de estudio se tomaron, durante 1998, estacionalmente, y en cada nivel, nueve unidades muestrales (u.m.) consistentes cada una en un cuadrado de $25 \mathrm{~cm}^{2}$, dispuestas en forma aproximadamente regular sobre dos transectas de $30 \mathrm{~m}$ de ancho, paralelas a la línea de costa, las que sumaron en conjunto 144 u.m., cubriendo con ellas la distribución vertical total de la población en la localidad, sobre sustrato rocoso. Para cada u.m. se registró la inclinación del sustrato como: vertical $\left(90^{\circ}\right)$, aproximada de $45^{\circ}$ y horizontal $\left(0^{\circ}\right)$.
Cada u.m. fue caracterizada individualmente a través del índice de radiación IR $\left(\mathrm{Mj} \mathrm{m}^{-2}\right.$ día $\left.^{-1}\right)$ que se elaboró tomando en cuenta la orientación de los sitios.

$$
I R_{S E N}=\sum_{i=1}^{3}\left(I L_{i} \times P_{i}\right)
$$

Donde: S, indica el sitio (I ó II); E, estación del año; N, el nivel (1 ó 2); $\mathrm{IL}_{\mathrm{i}}$ (con i = 1-3) el nivel de irradiación promedio correspondiente a los períodos de alta, media o baja luminosidad. $\mathrm{P}_{\mathrm{i}}$, la duración de estos períodos para los cual se dividió la longitud del día, tomada del almanaque náutico, en períodos de alta, media y baja radiación de acuerdo a los registros obtenidos in situ. La intensidad lumínica se midió en días sin nubosidad en cada estación de muestreo, desde la salida a la puesta del sol en cada sitio y cada nivel del intermareal, utilizando luxómetro TES-1330 digital. La radiación fotosintéticamente activa fue estimada de acuerdo a factores de transformación (McCree 1981, Oliveira et al. 1995).

Los datos de temperatura del aire y precipitaciones, y de la frecuencia relativa, velocidad media y ráfagas de los vientos fueron provistos por la oficina del puerto de Caleta Paula (Caleta Olivia, Provincia de Santa Cruz, Argentina).

La abundancia relativa de $B$. minima en cada u.m. fue calculada como la cantidad de individuos de esta especie por cada 100 talos del total de especies presentes. Para analizar las variaciones en las características de los individuos se tomaron al azar 100 talos dentro de cada u.m. y en cada uno se registró: presencia de ramificaciones, longitud expresada en una escala de 1 a 3 (1: talos < 0,19 cm; 2: entre 0,2 y 0,9 cm y 3: entre 1,0 y 5,0 cm) y el ordenamiento celular en tres porciones del talo. El ancho $(\mu \mathrm{m})$ y el grosor de la pared $(\mu \mathrm{m})$ del talo, se midieron en la porción media de 10 plantas de cada u.m. Los diámetros celulares $(\mu \mathrm{m})$ de 3 células se midieron en las porciones apical, media y basal de 7 talos de cada u.m.

En el sitio II, los talos se encontraron llenos de sedimento que no pudo ser eliminado completamente por lo que las determinaciones de biomasa y la observación de la fertilidad de los talos se vieron dificultadas de manera que solo se trabajó en estos aspectos en el sitio I.

B. minima forma céspedes en los que varios tubos erectos emergen de una costra basal de varias capas de células, por lo que se registró en cada u.m. el peso seco 
(a $100^{\circ} \mathrm{C}$ hasta peso constante) de un conjunto de talos y costras basales de B. minima. Los pesajes en laboratorio se realizaron con una balanza de análisis digital Bosch, S-2000/10/ STUDENT (Precisión: Max 200 g; Min 5 dg). Se compararon los valores promedio en peso seco de las unidades muestrales en ambos niveles.

La proporción estacional de plantas fértiles fue determinada por observación microscópica de ejemplares vivos recolectados en ambos niveles y en las cuatro estaciones (06-03-99; 16-04-99; 23-07-99; 2610-99); en cada oportunidad se recolectaron 100 talos $(\mathrm{n}=800)$. Para las mediciones y fotografías microscópicas se utilizó un fotomicroscopio Axioplan Zeiss D-7083, un esteromicroscopio Leica Wild Mb 8, un ocular micrométrico y equipo fotográfico Wild.

\section{Mediciones y métodos estadísticos}

Para constatar si los sitios seleccionados presentaban diferencias significativas en cuanto a la exposición al oleaje se analizó el desgaste de las esferas de yeso mediante un ANOVA de simple entrada. La heterogeneidad en los tamaños celulares de los talos y su variación estacional en peso seco, fueron tratadas con análisis de varianza.

Los datos del índice de irradiación (IR) fueron transformados para eliminar la influencia estacional y se analizó mediante ANOVA de doble entrada, para establecer diferencias significativas relacionadas con el nivel (1 y 2) y el sitio (I y II) de muestreo.

La variación estacional en peso seco por unidad de área, durante el período de estudio, se analizó mediante un ANOVA. Previamente se analizó la homogeneidad de varianzas y se procedió luego al correspondiente análisis de varianza. Éste se completó mediante una prueba a posteriori de Tukey y Kramer (Sokal \& Rohlf 1997).

La frecuencia de especimenes fértiles en diferentes estaciones del año y niveles del intermareal, se analizó aplicando un modelo log-lineal para tablas trifactoriales.

Para establecer asociación entre presencia de talos fértiles y estación del año, se efectuó un análisis de prueba de $\mathrm{G}$ para tablas $\mathrm{R}$ x C, ajustada por corrección de Williams, con los datos de cada nivel independientemente.
Los cálculos indicados se realizaron con el programa Biomstat (Sokal \& Rohlf 1981, Rohlf \& Slice 1997).

Para analizar los factores involucrados en la distribución de las especies intermareales, se realizó un análisis de redundancias (RDA) con el programa CANOCO, versión 4,0. Previamente al RDA, se llevó a cabo un análisis de correspondencias sin tendencias (DCA), el cual indicó que el largo de gradiente de los ejes de ordenación era menor o igual que uno, lo cual indica una distribución en gradiente antes que unimodal. Para este tipo de distribución, el método aconsejado por ter Braak (1998) es el RDA.

Los descriptores biológicos ingresados fueron los diámetros celulares promedio en la porción apical (D1), media (D2) y basal (D3) de los talos $(\mu \mathrm{m})$; longitud promedio de los talos (Lon) (mm); espesor medio de la pared (Gro) ( $\mu \mathrm{m})$; ancho en la parte media del talo (Anc) $(\mu \mathrm{m})$, proporción de plantas con ramificaciones (Ram) y abundancia relativa de $B$. minima respecto de otras especies (Bli) en una escala de 1 a 3 (1: <20\%; 2: 20 - 59\%; 3: >60\%). Los datos fueron relativizados al valor máximo. Las variables ambientales incluidas inicialmente en el análisis multivariado fueron: el grado de exposición al oleaje alto (SIT 1) o bajo (SIT 2); nivel en el intermareal alto (NIV 1) o bajo (NIV 2); el ángulo de inclinación del sustrato para cada muestra (INC); la temperatura promedio $\left({ }^{\circ} \mathrm{C}\right)$ mensual del aire (TAI); la temperatura promedio $\left({ }^{\circ} \mathrm{C}\right)$ del agua superficial (TAS); la longitud (minutos) del período luminoso (LPL); la velocidad del viento $\left(\mathrm{km} \mathrm{h}^{-1}\right)$ promedio, durante el mes anterior (VI1) y durante el mes de muestreo (VI2); las intensidades máximas del viento (RAF) durante el mes de muestreo $\left(\mathrm{Km} \mathrm{h}^{-1}\right)$; el promedio diario de las precipitaciones $(\mathrm{mm})$ del mes anterior (LL1) y las del mes de muestreo (LL2); la frecuencia (\%) mensual de los vientos del noreste, norte o este (VNE) y la frecuencia (\%) mensual de los vientos del sudoeste, oeste o sur (VSO); la irradiancia promedio medida in situ (joule $\mathrm{m}^{-2} \mathrm{~s}^{-1}$ ) (IRP) y el índice de irradiación diaria (Mjoules $\mathrm{m}^{-2}$ día $^{-1}$ ) para cada período estacional, sitio y nivel (IRD). Para evitar multicolinearidad fueron eliminadas las variables que presentaron valores de inflación de la varianza (VIF) superiores a 10 (TAS, LPL, VI1, LL2, IRP) y en la selección “forward”, las variables con valor de probabilidad > 0,05 (INC, RAF, VNE y LL1). Para el RDA se usaron las opciones estándar y para la matriz de correlación, centrada y estandarizada por variables biológicas, se utilizó un factor de escala doble ("scaling" = 2). 
Resultados

\section{Análisis preliminares al RDA}

Antes de proceder al análisis multivariado, se realizaron los siguientes análisis para definir las variables a utilizar, referidos, respectivamente, a la variabilidad en exposición al oleaje entre los sitios, a la homogeneidad de los diámetros celulares dentro del talo y al índice de irradiación.

Para constatar si los sitios seleccionados presentaban diferencias significativas en cuanto a la exposición al oleaje se analizó el desgaste de las esferas de yeso (Fig. 2) mediante un ANOVA. De acuerdo a este análisis se estableció que el sitio I (35,73 g) presenta un grado de exposición significativamente mayor $(\mathrm{F}=822,36$; $P<0,001)$ que el sitio II (0,52 g).

Las observaciones del diámetro celular en las tres porciones del talo responden al supuesto de una polaridad basal-apical frecuente en las Ulvaceae (Boraso 1975). La verificación mediante un ANOVA $(\mathrm{F}=59,76 ; P=0,001)$ de heterogeneidad en los tamaños celulares determinó su introducción en los análisis posteriores como variables separadas.

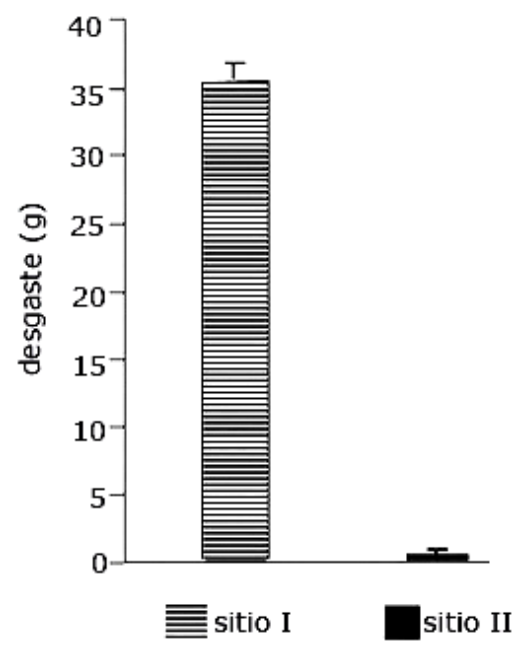

Figura 2

Desgaste de esferas de yeso (g) expuestas al oleaje durante $48 \mathrm{~h}$ en los dos sitios del estudio. Medias \pm desviación estándar (D.E.)

Wearing down of plaster spheres (g) exposed to the wave exposure during $48 \mathrm{~h}$, at both study sites. Average \pm standard deviation (S.D.)
En verano, los valores del índice son mayores que en el resto de las estaciones, en ambos niveles y sitios de estudio; en segundo término se ubican los valores del índice estacional obtenidos en primavera y luego los de otoño e invierno, respectivamente.

Sobre los datos del índice de irradiación corregidos para eliminar la estacionalidad, se constató que existen diferencias significativas con relación al nivel y el sitio. El índice de irradiación calculado es significativamente mayor $(P=0,017)$ en el sitio I que en el sitio II y, también es significativamente mayor $(P=0,019)$, en el nivel más bajo del intermareal comparado con el nivel más alto. No se halló interacción significativa entre sitios y niveles.

\section{Análisis de redundancias (RDA: en el diagrama biplot) (Fig. 3)}

Los descriptores ambientales se indican como vectores de líneas de puntos y los biológicos como vectores de líneas llenas. Quedaron definidos tres ejes que explican el 97,7\% de la variabilidad; los ejes I y II absorben respectivamente el $74,9 \%$ y el $14,7 \%$ de la relación factores biológicos - ambiente (Tabla 1).

En la Tabla 2 se muestran los valores de correlación pesada, la que relaciona los ejes de especies con las variables ambientales mencionadas. La ordenación de las muestras sobre el eje I indica que la proporción de $B$. minima y el grosor de la pared se relacionan con factores de naturaleza estacional (irradiación, temperatura del aire y vientos). El mayor grosor de la pared se asocia con temperaturas e irradiaciones bajas y los vientos del sudoeste. En la Fig. 4 se observa que durante el otoño, los talos de B. minima, ubicados en el nivel más bajo (2: 3,89-3,00 m), presentan paredes relativamente más gruesas.

La mayor abundancia relativa de talos de B. minima está correlacionada con temperaturas e índice de irradiación altos y con los meses en que los vientos SW, $\mathrm{S}$ y W fueron más intensos; en el muestreo de verano, la abundancia relativa de $B$. minima fue máxima, con valores de $70 \%$ o más en ambos sitios y niveles. El segundo eje describe una combinación de la temperatura y los factores locales asociados al sitio, con los que se correlacionan el diámetro celular, el ancho del talo y la presencia de ramificaciones en la base. El RDA indica que el aumento en el ancho de los talos está correlacionado con el incremento de la temperatura del aire $\left({ }^{\circ} \mathrm{C}\right)$, en primavera y verano, y secundariamente, 
con el índice de irradiancia. El ancho de los talos osciló entre 75 y $392 \mu \mathrm{m}$ durante el período de estudio; los talos fueron, en todas las estaciones, más anchos en el sitio menos expuesto al oleaje intenso; los talos más anchos se recolectaron en primavera y verano (Fig. 5).

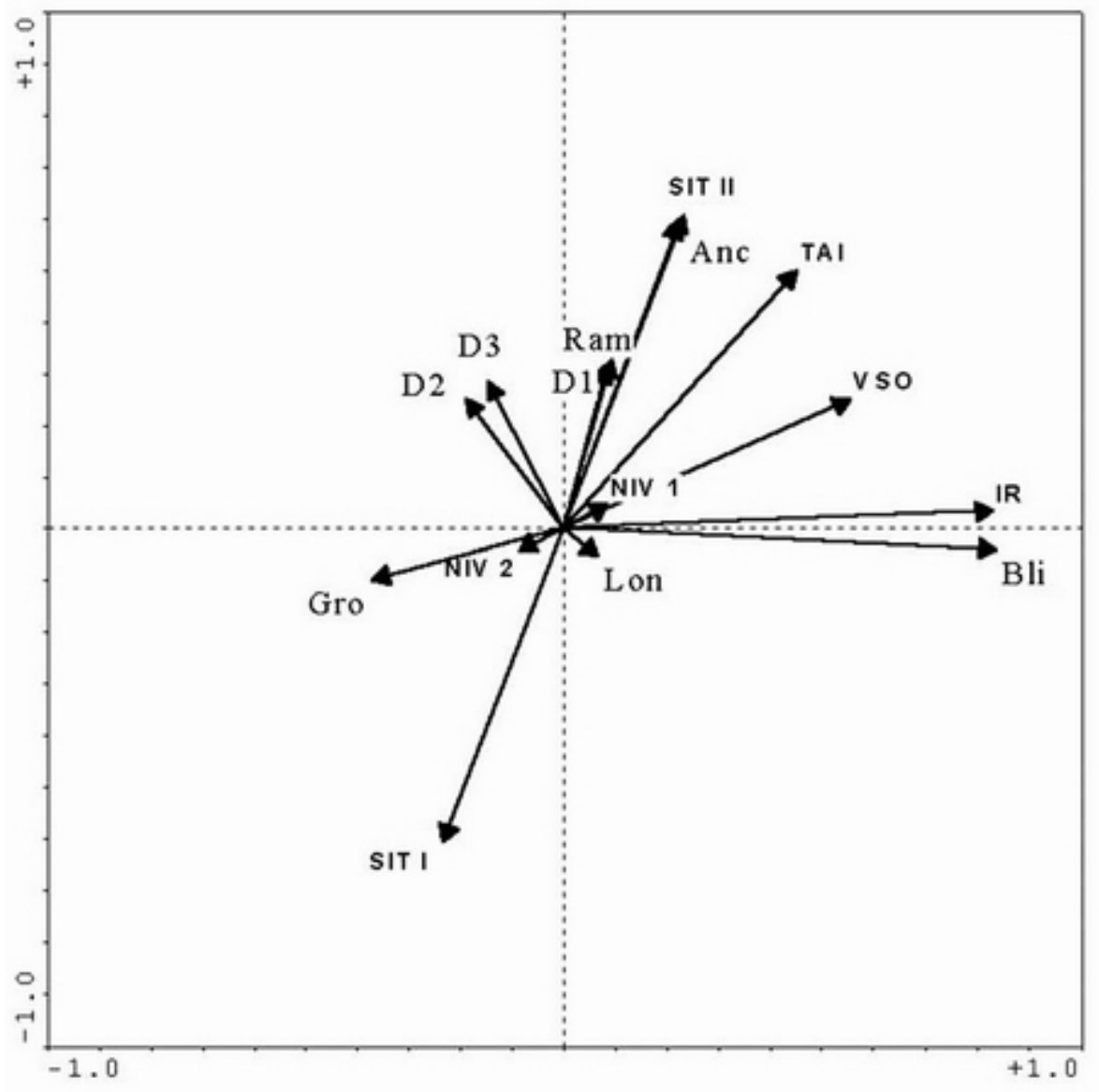

Figura 3

Diagrama (biplot) del análisis de redundancias. Descriptores biológicos: D1, D2 y D3, diámetro de las células en la porción apical, media e inferior del talo respectivamente; Lon, longitud promedio del talo; Gro, espesor de la pared; Anc, ancho del talo; Ram, presencia de ramificaciones; Bli, proporción(\%) de B. minima. Descriptores ambientales: SIT I, sitio con mayor grado de exposición al oleaje; SIT II, sitio con menor grado de exposición al oleaje; NIV 1: ubicación de las muestras en el sector más alto del intermareal; NIV 2: ubicación de las muestras en el sector más bajo del intermareal; TAI, la temperatura promedio mensual del aire $\left({ }^{\circ} \mathrm{C}\right)$; VSO, frecuencia en que los vientos soplaron del sudoeste; IRD, índice de irradiación estacional

Diagram (biplot) of the analysis of redundancies. Biological descriptors: D1, D2 and D3, cell diameter in the apical, middle and inferior portion of the thallus respectively; Lon, length average of thallus; Gro, wall thickness; Anc, thallus width; Ram, presence of branches; Bli, proportion (\%) of B. minima. Environmental descriptors: SIT I, site more exposed to waves; SIT II, site less exposed to waves; NIV 1: samples from the highest intertidal level; NIV 2: samples from the lowest intertidal level; TAI, monthly air average temperature $\left({ }^{\circ} \mathrm{C}\right)$; VSO, southwest winds frequency; IRD, seasonal irradiation index 
Tabla 1

Resumen de la ordenación por análisis de redundancias (RDA) de los factores ambientales y las características biológicas de B. minima en Punta Maqueda

Summary of the ordination by analysis of redundancies (RDA) of environmental factors and biological characteristics of B. minima in Punta Maqueda

\begin{tabular}{|c|c|c|c|c|c|}
\hline Ejes & 1 & 2 & 3 & 4 & $\begin{array}{c}\text { Inercia } \\
\text { total }\end{array}$ \\
\hline Valores propios: & 0,296 & 0,058 & 0,032 & 0,008 & 1,00 \\
\hline $\begin{array}{l}\text { Correlaciones variables biológicas — variables } \\
\text { ambientales: }\end{array}$ & 0,836 & 0,632 & 0,395 & 0,316 & \\
\hline $\begin{array}{l}\% \text { acumulado de la varianza de los datos de variables } \\
\text { biológicas: }\end{array}$ & 29,60 & 35,40 & 38,60 & 39,40 & \\
\hline $\begin{array}{l}\% \text { acumulado de la varianza de la relación variables } \\
\text { biológicas — variables ambientales: }\end{array}$ & 74,90 & 89,60 & 97,70 & 99,70 & \\
\hline Suma de los valores propios no restringidos & & & & & 1,00 \\
\hline Suma de todos los valores propios canónicos & & & & & 0,395 \\
\hline
\end{tabular}

Tabla 2

Matriz de correlación pesada entre los ejes de variables biológicas y variables ambientales

Weighted correlation matrix of the biological variables axes with environmental variables

\begin{tabular}{ccccc}
\hline Nombre & Eje 1 & Eje 2 & Eje 3 & Eje 4 \\
\hline SIT I & $-0,1941$ & $-0,3834$ & $-0,1119$ & $-0,1699$ \\
SIT II & 0,1941 & 0,3834 & 0,1119 & 0,1699 \\
NIV 1 & 0,0705 & 0,0287 & $-0,3585$ & 0,1315 \\
NIV 2 & $-0,0705$ & $-0,0287$ & 0,3585 & $-0,1315$ \\
TAI & 0,3764 & 0,3154 & 0,0067 & $-0,0423$ \\
VSO & 0,4646 & 0,1592 & $-0,0962$ & $-0,2173$ \\
IRD & 0,6943 & 0,0208 & 0,0431 & $-0,0446$ \\
\hline
\end{tabular}

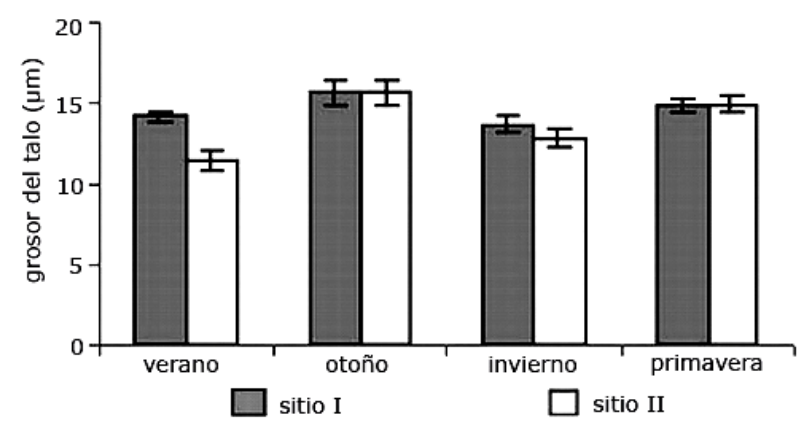

Figura 4

Variación estacional del grosor de la pared del talo $(\mu \mathrm{m})$ de B. minima. Sitio I: mayor exposición al oleaje; Sitio II: menor exposición al oleaje. Promedio \pm error estándar

Seasonal variation of the thallus wall thickness $(\mu \mathrm{m})$ of $B$. minima. Site I: more wave exposure; Site II: less wave exposure. Average \pm standard error 


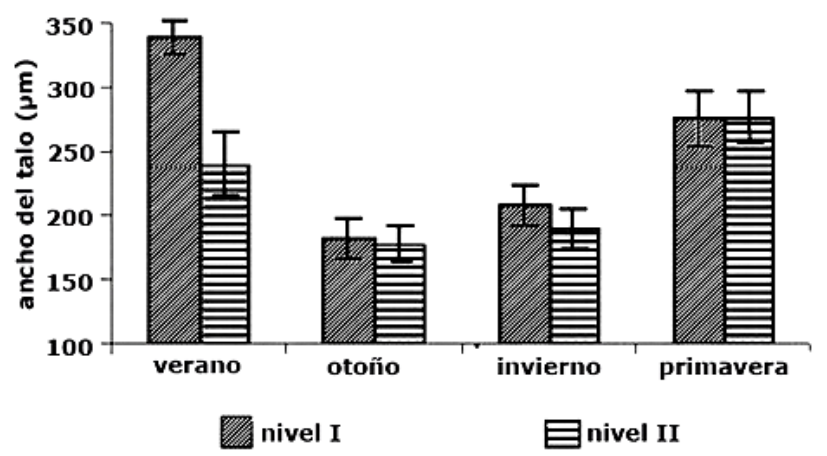

Figura 5

Variación estacional del ancho $(\mu \mathrm{m})$ del talo de $B$. minima de los dos niveles intermareales. Promedio \pm error estándar

Seasonal variations of thallus width $(\mu \mathrm{m})$ in B. minima from the two intertidal levels. Average \pm standard error

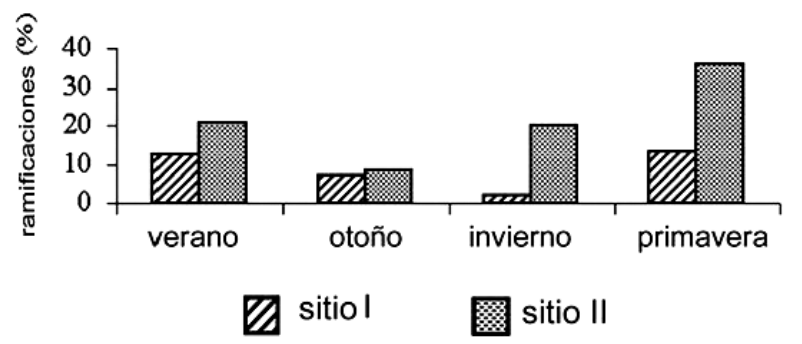

Figura 6

Variación estacional en la proporción de plantas con ramificaciones de $B$. minima. Sitio I: mayor exposición al oleaje; Sitio II: menor exposición al oleaje

Seasonal variation in the proportion of talli with ramifications of B. minima. Site I: more wave exposure; Site II: less wave exposure

La proporción de talos con ramificaciones fue mayor en el sitio menos expuesto al oleaje (II). En los dos sitios se observa el mismo perfil de variación estacional en la ramificación (Fig. 6).

El RDA indica que el diámetro celular promedio en las tres zonas, está relacionado con el aumento de la temperatura del aire y el sitio menos expuesto al oleaje (D1, D2, y D3: Fig. 3). La longitud de los talos se asocia con el tercer eje definido principalmente, por los niveles del intermareal considerados; las longitudes mayores (LON) se hallan asociadas al nivel más bajo del intermareal (NIV2) siendo esta relación más evidente en otoño, en el sitio I (Fig. 7).

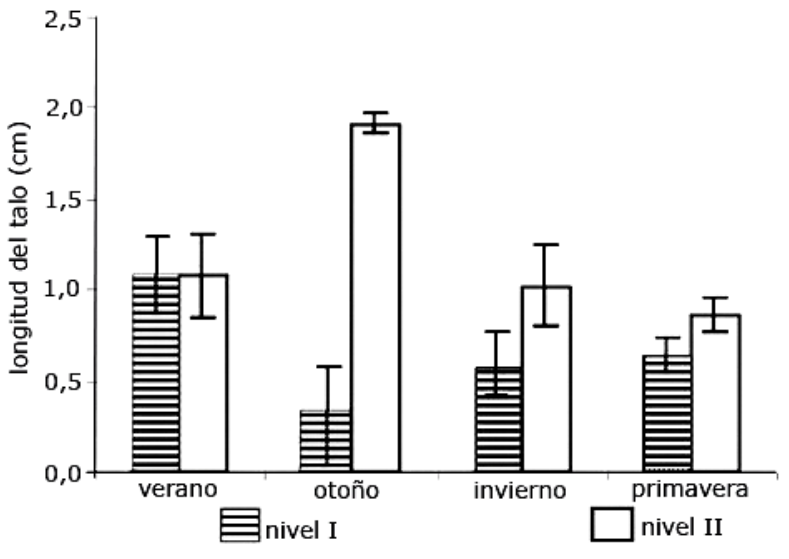

Figura 7

Variación estacional en la longitud de talos de $B$. minima (cm) en dos niveles intermareales (sitio I). Promedio \pm error estándar

Seasonal variation in the length of $B$. minima thalli $(\mathrm{cm})$ from two intertidal levels (site I). Average \pm standard error

\section{Variación estacional de la biomasa}

El valor promedio en peso seco por unidad de superficie en el sitio I fue de $256 \mathrm{~g} \mathrm{~m}^{-2}$ en verano, de $276 \mathrm{~g} \mathrm{~m}^{-2}$ en primavera, de $188 \mathrm{~g} \mathrm{~m}^{-2}$ en invierno y de $104 \mathrm{~g} \mathrm{~m}^{-2}$ en otoño. La variación estacional de la biomasa tuvo un valor de $F$ significativo $(\mathrm{F}=4,34 ; \quad P=<0,01)$, la correspondiente prueba a posteriori, indicó que la biomasa de $B$. minima, en primavera, fue significativamente mayor $(P<0,01)$ que en otoño, mientras que las diferencias con el resto de las estaciones no fueron significativas (Tabla 3).

\section{Tabla 3}

Comparación estacional de la biomasa de B. minima (peso seco, $\mathrm{g} \mathrm{m}^{-2}$ ): Prueba de Tukey \& Kramer. $(* *) P<0,01$

Seasonal comparison of the biomass of $B$. minima (dry weight, $\mathrm{g} \mathrm{m}^{-2}$ ): Tukey \& Kramer test. $\left({ }^{* *}\right) P<0,01$

\begin{tabular}{ccccc}
\hline & verano & otoño & invierno & primavera \\
\hline verano & - & 153,778 & 67,556 & 19,556 \\
otoño & - & - & 86,222 & $173,333^{* *}$ \\
invierno & - & - & - & 87,111 \\
\hline
\end{tabular}




\section{Influencia del nivel mareal y estación del año sobre la fertilidad}

La proporción de talos fértiles (A) en cada nivel del intermareal (B) y cada estación de año (C) fue analizada utilizando un modelo log-lineal para tablas trifactoriales. Al no existir indicación de una interacción trifactorial entre A (fertilidad), B (nivel del intermareal) y C (estación del año) ( $\mathrm{G}_{\mathrm{ABC}}$ no significativo), el modelo probado ajustó a los datos observados y se procedió a analizarlos mediante modelos jerárquicamente más simples, concluyéndose que la fertilidad (A) depende tanto del nivel (B) como de la estación del año (C) y varía igualmente en los distintos niveles del intermareal para las diferentes estaciones. La proporción de talos fértiles por nivel del intermareal en conjunto, fue mayor en el nivel más alto que en el nivel más bajo durante todo el período de estudio. Para verificar si existía asociación significativa entre la presencia de talos fértiles y alguna estación del año en particular, se realizaron análisis para tablas $\mathrm{R}$ x $\mathrm{C}$ con los datos de cada nivel por separado, verificando que existe una asociación altamente significativa entre la estacionalidad y la fertilidad de talos ( $\mathrm{G}=388,69$; $P<0,01 ; \mathrm{GL}=3$ ). La frecuencia de talos fértiles fue significativamente mayor en otoño y primavera, con relación a verano e invierno, tanto en el nivel 1 , $(\mathrm{G}=210,47 ; \quad P<0,01 ; \quad \mathrm{GL}=3) \quad$ como en el nivel 2 ( $\mathrm{G}=190,29 ; P<0,01 ; \mathrm{GL}=3)$ (Fig. 8).

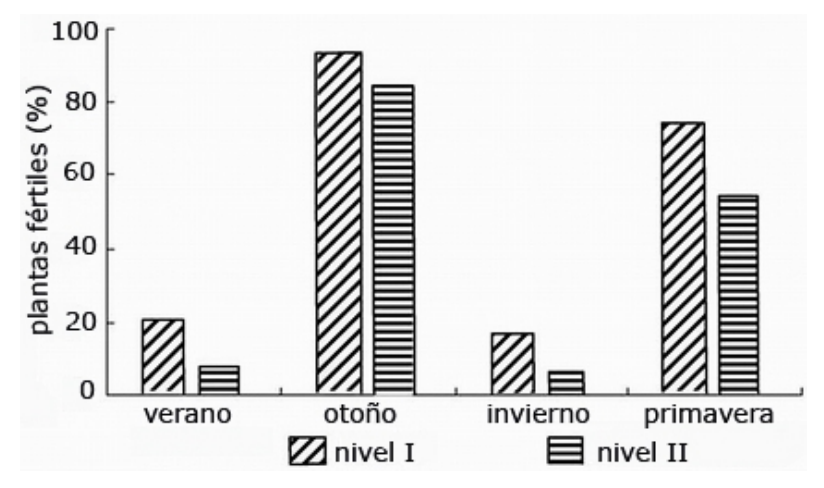

Figura 8

Variación estacional de la proporción de talos fértiles de $\boldsymbol{B}$. minima $(\mathrm{n}=100)$ de los dos niveles intermareales del estudio (sitio I)

Seasonal variation in the proportion of fertile $B$. minima thalli $(n=100)$ from the two studied intertidal levels (site I)

\section{Discusión y conclusiones}

Numerosos autores entre los que se puede mencionar a Chamberlain (1965), Munda \& Markham (1982) y Sohn (1983) a nivel mundial, y Boraso (1970), Zaixso \& Pastor (1977), Zaixso et al. (1978) y Boraso \& Zaixso (1996) para Argentina, señalan que $B$. minima se desarrolla preferentemente en la zona más alta del intermareal; sin embargo, Kepel (1999) la indica como especie dominante en el intermareal medio y Boraso de Zaixso (2004) señala su presencia en los niveles altos e intermedios del intermareal. En la etapa del estudio exploratorio del presente trabajo se observó una distribución relativamente amplia de la especie abarcando ambos niveles, aunque solo se presenta en bandas continuas en los niveles más altos donde la competencia con otras especies sería aparentemente menor.

Tittley (1982) observó en el área de Woolwich (U.S.A.) que, tanto en verano como en invierno, $B$. minima ocupó niveles altos por sobre una banda de Rhizoclonium riparium. Las variaciones en la zonación fueron atribuidas a diferencias en el tipo de hábitat como la dominancia de $B$. minima en paredes de concreto y su reemplazo por Rhodochorton purpureum (como Audouinella purpurea) en sitios de sombra; este reemplazo se observa también en Punta Maqueda, en el intermareal superior, dentro de cuevas.

Edwards (1977) analizó, en laboratorio, el crecimiento de varias especies de algas, encontrando poca correlación entre el nivel que ocupan en el intermareal y su capacidad de crecimiento con diferentes grados de exposición al aire, creciendo todas mejor a una inmersión del 100\%. Esto apoya la hipótesis de que las algas del intermareal han sufrido exclusión competitiva del hábitat más favorable y no tienen rechazo a las condiciones hostiles del intermareal. Sin embargo, de acuerdo con Tittley (1982) existirían especies de algas intermareales que, por necesidades metabólicas reales o por efectos secundarios de la inmersión continua, como anoxia o infecciones bacterianas, requieren realmente de exposiciones periódicas. Según Prange (1978), el límite superior de la distribución vertical de Blidingia minima var. subsalsa (Kjellman) Scagel estaría asociado a condiciones osmóticas desfavorables, por ejemplo, lluvias intensas o desecación extrema y, el límite inferior, a bajas intensidades lumínicas. Dromgoole (1980) observó que el sombreo, la protección al oleaje y la presencia de vegetación modifican significativamente 
la tasa de desecación de los talos individuales de varias especies. En nuestro estudio, hallamos a B. mínima colonizando los niveles superiores del intermareal rocoso en un sector afectado por el sombreo del acantilado. Sin embargo, observamos que en niveles intermareales medios crece tanto sobre talos de Porphyra columbina, una especie fotófila, como sobre Stictosiphonia intricata, una especie umbrófila, por lo cual se podría inferir que no es la luz lo que determina su límite de distribución inferior.

Prange (1978), Thom (1984), Munda \& Markham (1982), Kepel (1999) Ballesteros (1991) y nuestros resultados en Punta Maqueda señalan una mayor abundancia de Blidingia en los meses estivales y de primavera.

La acción de los vientos puede ser negativa para la biomasa in situ de algunas especies causando su deprendimiento, o puede ser beneficiosa al promover movimientos del agua que aportan nutrientes. Esta última podría ser la situación de $B$. minima por la relación que hay entre los vientos más intensos del sector sur y la mayor proporción de la especie en las muestras. Se ha indicado la preferencia de $B$. minima por lugares expuestos a turbulencia del agua (Chamberlain 1965) y por regímenes de corrientes intensas (Mathieson et al. 1983).

A diferencia de la información sobre las variaciones espaciales o temporales de su abundancia la información referida a la influencia del ambiente sobre las características morfológicas, anatómicas y de la reproducción de $B$. minima en los ambientes naturales es muy escasa. Varias de las características biológicas analizadas en el presente estudio se relacionaron con características ambientales locales como la exposición al oleaje y el sombreado en cada sitio. Bliding (1963) analiza, en forma combinada, los efectos del nivel y exposición al oleaje, indicando que sobre el litoral superior, los talos de B. minima no superan los $2 \mathrm{~cm}$ de longitud $y$ son muy angostos, mientras que en localidades protegidas alcanzan más de $24 \mathrm{~cm}$ de alto y hasta $4 \mathrm{~mm}$ de diámetro. En el golfo San Jorge en cambio, los efectos de nivel y exposición al oleaje sobre el tamaño y forma se separan claramente, observándose que el ancho de los talos respondía a la exposición y la longitud al nivel del intermareal, encontrándose talos más largos en niveles más bajos, especialmente durante el otoño.
En coincidencia con las observaciones sobre esta especie de Bliding (1963) y Boraso (1975), la presencia de ramas es escasa. Las plantas con alguna rama se encontraron, preferentemente, en épocas de temperaturas relativamente altas y en el sitio con menos turbulencia.

Los valores máximos en el diámetro celular se hallan asociados, en todo el talo, a valores de baja movilidad del agua, sugiriendo que en esas condiciones la división celular podría ser más lenta.

Muchos organismos responden a las situaciones de estrés incrementando su esfuerzo reproductivo (Pringle 1986, Eyras \& Boraso de Zaixso 1994). La frecuencia de talos fértiles de $B$. minima fue mayor durante todo el período de estudio en Punta Maqueda, en el nivel más alto del intermareal, donde se encuentran sujetos a mayor estrés por desecación que en los inferiores.

Por otra parte, la frecuencia de talos fértiles fue más alta en otoño y primavera, que en verano e invierno. La relación de la fertilidad con la estacionalidad podría deberse a factores estrictamente estacionales como luz y temperatura pero no se puede descartar la influencia de la concentración de nutrientes que suele presentar variaciones temporales.

La población estudiada de Blidingia minima se mantuvo durante todo el año, observándose diferencias significativas en la biomasa solo entre los máximos de primavera y los mínimos observados en otoño. Durante primavera aumenta también el diámetro de las células y el tamaño promedio de los talos, así como la proporción de talos con ramificaciones y comienza también a incrementarse la proporción de talos fértiles. En el verano, la biomasa es intermedia, los individuos son más anchos y de mayor longitud, con alguna ramificación basal y paredes delgadas en el nivel más bajo del intermareal. En otoño, la población estuvo constituida mayoritariamente por un césped corto de talos de paredes gruesas, se observó la máxima frecuencia de talos fértiles, lo que no se tradujo sin embargo, en un evidente aumento de biomasa en la siguiente estación. Es probable, por lo tanto, que una de las formas en que la especie pase el invierno sea como talos microscópicos y porciones basales, hasta la siguiente primavera, en que se reinicia el crecimiento de las porciones erectas. 
La distribución de B. minima va desde el Caribe hasta la Antártida (Bliding 1963, Scagel 1966, Papenfuss 1955, Norris 1971, Kornmann \& Sahling 1978, Garbary \& Barkhouse 1987, Laturnus 1996, Harper \& Garbary 1997, Gallardo et al. 1999, Kepel 1999) lo que permite inferir una diversidad de situaciones ambientales en las que puede haber desarrollado, por un lado, plasticidad fenotípica y, por otro, diferenciación genética, como la detectada en las costas de Japón por Woolcott et al. (2000). Dentro de un área muy reducida en Punta Maqueda, obviamente homogénea respecto al clima y de la masa de agua, los resultados obtenidos relacionan la variabilidad morfológica de esta especie con la estacionalidad, el nivel en el intermareal, la iluminación y la exposición al oleaje.

Para la comprensión de las estrategias de esta especie en relación con los factores ambientales quedan por profundizar aspectos del reclutamiento y de la propagación clonal, cuya importancia para las algas marinas ha sido señalada por Collado-Vides (2001).

\section{Agradecimientos}

Agradecemos al Lic. Héctor Zaixso por su asesoramiento en los análisis estadísticos. A los Dres. Néstor Cazzaniga y Atila Gosztonyi por el análisis crítico y sus valiosas sugerencias y a los tres evaluadores anónimos que contribuyeron a mejorar nuestro documento. Al personal del puerto de Caleta Paula (Caleta Olivia, Provincia de Santa Cruz), por los registros de datos ambientales del periodo de estudio.

\section{Literatura citada}

Ballesteros E. 1991. Structure and dynamics of the community of Rissoella verruculosa (Bertoloni) J. Agardh (Gigartinales, Rhodophyceae) in the northwestern Mediterranean. Scientia Marina 55 (2): 439-51.

Bliding C. 1938. Studien über Entwicklung und Systematik in der Gattung Enteromorpha I. Botaniska Notiser 1938: 8390 .

Bliding C. 1963. A critical survey of European taxa in the Ulvales. Part. I. Capsosiphon, Percusaria, Blidingia, Enteromorpha. Opera Botánica 8 (3):1-160.

Boraso A. 1970. Sobre la presencia de Blidingia minima (Nägeli ex Küzing) Kylin var. minima en la Argentina. Physis 30 (80): 167-170.
Boraso A. 1975. Los géneros Enteromorpha, Blidingia y Percusaria (Algae, Chlorophyta) en las costas atlánticas argentinas. Darwiniana 19: 85-311.

Boraso de Zaixso AL. 2004. Chlorophyta marinas de Argentina. Historia Natural, (Bs. As.) Segunda Serie 3 (11): 95-119.

Boraso AL \& HE Zaixso. 1996. Asociaciones de macroalgas intermareales en el golfo San José (Provincia del Chubut, Argentina). Naturalia Patagónica 4 (1-2): 47-64.

Chamberlain YM. 1965. Marine algae of Gough Island. Bulletin of the British Museum (Natural History), Botany Series 3: 173-232.

Collado-Vides L. 2001. Clonal architecture in marine macroalgae: ecological and evolutionary perspectives. Evolutionary Ecology 15 (4-6): 531-545.

Doty MS. 1971. Measurement of water movement in reference to benthic algal growth. Botánica Marina 14: 3235.

Dromgoole FI. 1980. Desiccation resistance of intertidal and subtidal algae. Botánica Marina 23: 149-159.

Eyras MC \& AL Boraso de Zaixso. 1994. Aspectos de la estrategia reproductiva de Macrocystis pyrifera (Phaeophyta, Laminariales) en poblaciones de la costa argentina, Naturalia Patagónica, Ciencias Biológicas 2 (12): 33-47.

Edwards P. 1977. An investigation of the vertical distribution of selected benthic marine algae with a tide-simulating apparatus. Phycology 13: 62-8.

Gallardo T, IM Pérez Ruzafa, A Flores Moya \& F Conde. 1999. New collections of benthic marine algae from Livingston and Deception Islands (South Shetland Islands) and Trinity Island (Bransfield Strait) Antarctica. Botánica Marina 42 (1): 61-69.

Garbary D \& LB Barkhouse. 1987. Blidingia ramifera (Bliding) stat. nov. (Chlorophyta): a new marine algae for eastern North America. Nordic Journal of Botany 7: 359363.

Gerard VA \& KH Mann. 1979. Growth and production of Laminaria longicruris (Phaeophyta) populations exposed to different intensities of water movement. Phycology 15: 33-4.

Harper JT \& DJ Garbary. 1997. Marine Algae of Northern Senegal: the flora and its biogeography. Botánica Marina 40: 129-138. 
Hayden HS, J Blomster, Ch A Maggs, PC Silva, MJ Stanhope \& JR Waaland. 2004. Linnaeus was right all along: Ulva and Enteromorpha are not distinct genera. European Journal of Phycology 38: 277-294.

Karsten U. 1991. Ecophysiological investigation on the salinity and temperature tolerance of Antarctic green algae with emphasis of beta-metabolism. Berichte zur Polarforschung/Reports on Polar Research 79: 108

Karstens U, C Wiencke, G Kirst. 1992. Dimethylsulphoniopropionate (DMSP) accumulation in green macroalgae from polar to temperate region interactive effects of light versus salinity and light versus temperature. Polar Biology 12 (6-7): 603-607.

Kepel AA. 1999. Seasonal dynamics of intertidal macrophytobenthos in the mouth of channel of Lake Ptichye (Southwestern Peter the Great Bay, Sea Japan). Russian Journal of Marine Biology 25(5): 382-387.

Kornmann P \& PH Sahling. 1978. The Blidingia - species of Helgoland (Ulvales, Chlorophyta). Helgoländer wissenschaftliche Meeresuntersuchungen 31 (4): 391-413.

Kylin H. 1947. Über die Fortpflanzungsverhältnisse in der Ordnung Ulvales. Kungl. Fysiografiska Sällskapets Lund Förhandlingar 17: 174-182.

Larsen A \& K Sand Jensen. 2006. Salt tolerance and distribution of estuarine benthic macroalgae in the Kattegat-Baltic Sea area. Phycologia 45 (1): 13-23.

Laturnus F. 1996. Volatile halocarbons released from Arctic macroalgae. Marine Chemistry 55 (3-4): 359-366.

Mathieson AC, CD Neefus \& C Emerich Penniman. 1983. Benthic ecology in an estuarine tidal rapid. Botánica Marina 26 (5): 213-230. (Contribution No 1225 from Agricultural Experiment Station Series)

McCree KJ. 1981. Photosynthetically active radiation. En: Lange OL, PS Nobel, CB Osmond \& H Ziegler (eds), Encyclopedia of Plant Physiology, New Series 12: 41-55.

Munda IM \& JM Markham. 1982. Seasonal variations of vegetation patterns and biomass constituents in the rocky eulittoral of Helgoland. Helgoländer Meeresuntersuchungen 35 (2): 131-151.

Norris JN. 1971. Observations on the genus Blidingia (Chlorophyta) in California. Journal of Phycology 7: 145149.
Oliveira E, E Paula, E Plastino \& R Petti. 1995. Metodologías para cultivo no axénico de macroalgas marinas in vitro. En: Alveal K, ME Ferrario, EC Oliveira \& E Sar (eds). Manual de Métodos Ficológicos. pp. 430447. Universidad de Concepción, Chile.

Papenfuss G. 1955. Classification of the algae. In a century of progress in the natural sciences. California Academy of Sciences, San Francisco: 1853-1953.

Prange RK. 1978. An autoecological study of Blidingia minima var. subsalsa (Chlorophyceae) in the Squamish estuary (British Columbia). Canadian Journal of Botany 56: $170-179$

Pringle JD. 1986. Swarmer release and distribution of lifecycle phases of Enteromorpha intestinalis (L.) (Chlorophyta) in relation to environmental factors. Journal of Experimental Marine Biology and Ecology 100: 97111.

Rohlf FJ \& DE Slice. 1997. BIOMstat for Windows: Statistical software for Biologists. Version 3:2 c. Exeter Software (N.Y.), 61 pp.

Scagel RF. 1966. Marine algae of British Columbia and Northern Washington, Part I: Chlorophyceae (green algae). Bulletin of the National Museum of Canada 207, $257 \mathrm{pp}$

Servicio de Hidrografia Naval. 1998. Tablas de Marea. Publicación Servicio Hidrográfico Naval, Armada Argentina, $474 \mathrm{pp}$.

Servicio de Hidrografia Naval. 1999. Tablas de Marea. Publicación Servicio Hidrográfico Naval, Armada Argentina, 467 pp.

Sokal RR \& FJ Rohlf. 1981. Biometry, the principles and practice of statistics in biological research. $2^{\text {nd }}$ edition. 859 pp. New York. Freeman WH \& Company.

Sokal RR \& FJ Rohlf. 1997. Biometry, the principles and practice of statistics in biological research. $3^{\text {rd }}$ edition. 887 pp. New York. Freeman WH \& Company.

Sohn CH. 1983. A study on the algal communities of Odongdo, southern coast of Korea. Bulletin of the Korean Fisheries Society 16 (4): 368-378.

ter Braak CJF. 1998. CANOCO, a program for canonical community ordination by partial, detrended, and canonical correspondence analysis, principal component analysis and redundancy analysis (version 4.0). Centre for Biometry Wageningen, Wageningen, The Netherlands, 34 pp. 
Tittley I. 1982. Algal studies in the brackish reaches of the river Thames. British Phycological Society Winter Meeting, London (UK). Phycology 17 (2): 240.

Thom RM. 1984. Composition, habitats, seasonal changes and productivity of macroalgae in Grays Harbor Estuary, Washington. Estuaries 7 (1): 51-60.

Woolcott GW, M Iima \& RJ King. 2000. Speciation within Blidingia minima (Chlorophyta) in Japan: Evidence from morphology, ontogeny, and analyses of nuclear rDNA its sequence. Journal of Phycology 36 (1): 227-236.
Zaixso HE \& CT Pastor. 1977. Observaciones sobre la ecología de los mitílidos de la ría Deseado. I. Distribución y análisis biocenótico. Ecosur, Argentina 4: 1-46.

Zaixso HE, AL Boraso de Zaixso \& JJ Lopez Gappa. 1978. Observaciones sobre el mesolitoral rocoso de la zona de Ushuaia (Tierra del Fuego, Argentina). Ecosur, Argentina, 5 (10): 119-130. (Contribución $\mathrm{N}^{\circ} 156$ Centro de Investigaciones de Biología Marina)

Recibido el 24 de junio de 2005 y aceptado el 19 de enero de 2006 